\title{
Dual-angle optical coherence tomography for index of refraction estimation using rigid registration and cross-correlation
}

Christos Photiou

Costas Pitris 


\title{
Dual-angle optical coherence tomography for index of refraction estimation using rigid registration and cross-correlation
}

\author{
Christos Photiou and Costas Pitris* \\ University of Cyprus, KIOS Research and Innovation Center of Excellence, Department of Electrical and Computer Engineering, Nicosia, Cyprus
}

\begin{abstract}
The index of refraction ( $n$ ) of materials and/or tissues depends on their physical properties and serves as a source of optical contrast in imaging. The variations of the index of refraction have also been investigated for diagnostic purposes in various fields, such as hematology, oncology, etc., since they can signify disease and cell dynamic changes. Optical coherence tomography (OCT) has been used in the past to measure the index ex vivo. However, most methodologies described in the literature are not appropriate for in vivo imaging since they require either a mirror below the sample or a complicated imaging setup and algorithms. We describe a technique that uses two images, obtained at different angles, to estimate the index of refraction and can, thus, also be applied in vivo. The index of refraction is calculated from the path-length difference observed by the OCT beam at the two different angles. When a reflector is not available, the path-length difference can be estimated using image registration and the cross-correlation of adjacent $A$-scans. The proposed technique was validated experimentally using both clear and scattering samples. The resulting values of the index of refraction were within $\sim 1 \%$ of the expected. The main limitation of this technique is the effect of misalignment on the results, requiring the precision provided by an angular-resolved OCT system. These very promising results provide evidence that the dual-angle method should be further investigated and validated on human tissues so that it can be developed into a clinically useful diagnostic tool in the future. () The Authors. Published by SPIE under a Creative Commons Attribution 4.0 Unported License. Distribution or reproduction of this work in whole or in part requires full attribution of the original publication, including its DOI. [DOI: 10.1117/1.JBO.24.10.106001]
\end{abstract}

Keywords: optical coherence tomography; refractive index; image processing; cross-correlation; algorithm.

Paper 180683R received Dec. 27, 2018; accepted for publication Aug. 6, 2019; published online Oct. 4, 2019.

\section{Introduction}

The index of refraction $(n)$ is an important intrinsic optical parameter of tissue that has been recently exploited for various applications. All materials have unique $n$ values, which are associated with their electrical permittivity. Medical imaging systems use $n$ as a source of optical imaging contrast to view tissue without the need of labeling or extrinsic contrast agents. Studies have shown that the distribution of $n$ in tissue, using data from medical systems such as computed tomography and optical coherence tomography (OCT), could also be used as a biomarker for medical diagnosis. ${ }^{1,2}$ Microbiology, hematology, and infectious disease studies show that $n$ distribution can provide valuable information about cellular growth and division and bacteria identification. ${ }^{3-5}$ Various methods exist for the measurement of $n$ of different materials including tissues. ${ }^{6-8}$

Using OCT, a common method to estimate $n$ is based on measuring the path-length change from a sample placed over a reflector. ${ }^{9}$ Although this method is only appropriate for ex vivo measurements, its accuracy and simplicity make it ideal for the verification of newly developed techniques. This method exploits the fact that the optical path-length measured by OCT is proportional to the index of refraction. Thus, measuring the same distance in air and under a known thickness of tissue $(L)$, the index of refraction can easily be calculated as $n=$ $\left(L+L^{\prime}\right) / L$ (Fig. 1).

\footnotetext{
*Address all correspondence to Costas Pitris, E-mail: cpitris@ucy.ac.cy
}

Other methods that have been proposed depend on very complicated experimental setups and algorithms and are also very hard to apply in vivo. They either require specific hardware to precisely track the focus and calculate the path length and/ or require iterative methods to calculate the index of refraction by fitting to assumed models, which are processing-intensive and time-consuming. Focus tracking method and optical path shifting methods were evaluated for simultaneously calculating refractive index and thickness, in samples with different scattering properties. These methods required specialized hardware and multiple measurements. In addition, the optical path shifting method is limited to in vitro measurements albeit at a high precision. ${ }^{10}$ Gradient refractive index profile was also retrieved by iterative fitting of the optical path calculated by the ray tracing method, with that experimentally measured using OCT in the isolated crystalline fish eye lens. ${ }^{11}$ Further studies have demonstrated the calculation of the index of refraction of isolated mouse crystalline lens to determine whether index changes occur across age, due to gene mutations or with form deprivation myopia. ${ }^{12,13}$ Angularly resolved low-coherence interferometry to measure the phase index of refraction of pure silica by taking various thickness measurements at different incidence angles was also demonstrated but was only applied to nonbiological samples. ${ }^{14}$ Recently, a new study formulated a new method, using FD-OCT measured optical path lengths (OPLs) and properly selected spectral components of FD-OCT interference spectrum, to simultaneously measure path length and index of refraction. ${ }^{15}$ However, this method has not been demonstrated in vivo and the authors expressed concerns regarding the detrimental effects of speckle and beam aberrations caused by tissue. 


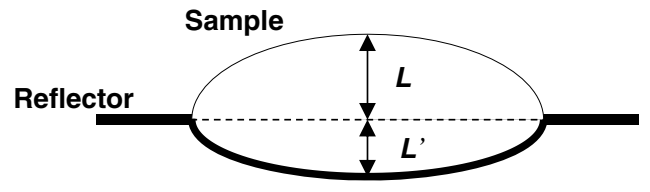

Fig. 1 Index of refraction calculation based on the path-length difference observed in the OCT imaging of samples with different indices of refraction. ${ }^{9}$

Even more recently, a refractive index correction algorithm that can find and correct the refractive index of all layers in a multilayer tissue, simultaneously, was also proposed. This algorithm can exploit parallel processing hardware thus achieving short processing times and is not affected by speckle. ${ }^{16}$ This technique was demonstrated on phantoms and skin tissue. A new method, appropriate for in vivo and in situ measurement of $n$ in tissues, without the need for a reflector as a reference, with minimal postprocessing and hardware modifications, is proposed here. Such a technique could be developed into a useful in vivo diagnostic tool in the future.

\section{Theory and Methods}

\subsection{Theory}

The proposed technique is applicable to both scattering, e.g., tissue, and nonscattering, e.g., glass, samples. When a sample, of any kind, is horizontal, the light is incident on the glass perpendicularly and reaches the bottom of the sample without changing the direction. The OPL $\left(L_{1}\right)$ from the top to the bottom surface of the sample is equal to the sample thickness $(d)$ multiplied by the index of refraction $(n)$. If the incidence is at an angle, the beam is refracted and, as it passes through the medium, it follows a path length $\left(L_{2}\right)$ that is longer than that of perpendicular incidence [Fig. 2(a)].

Given that the path lengths and incidence angles can be measured from the OCT images, the index of refraction can be estimated, using Snell's law's, as

$$
\left.\begin{array}{c}
\sin \theta_{1,2}=n \sin \theta_{2,2} \Rightarrow n=\frac{\sin \theta_{1,2}}{\sin \theta_{2,2}} \\
\left.\begin{array}{c}
L_{1}=n d \\
L_{2}=\frac{n d}{\cos \theta_{2,2}}
\end{array}\right\} \Rightarrow \frac{L_{1}}{L_{2}}=\cos \theta_{2,2} \Rightarrow \theta_{2,2}=\cos ^{-1}\left(\frac{L_{1}}{L_{2}}\right)
\end{array}\right\} \Rightarrow
$$

If the beams in both images are at an angle [Fig. 2(b)], the equations become:

$$
\begin{aligned}
& \sin \theta_{1,1}=n \sin \theta_{2,1} \Rightarrow \sin \theta_{2,1}=\frac{\sin \theta_{1,1}}{n} \\
& \left.\begin{array}{c}
\sin \theta_{1,2}=n \sin \theta_{2,2} \Rightarrow \sin \theta_{2,2}=\frac{\sin \theta_{1,2}}{n} \\
L_{1}=\frac{n d}{\cos \theta_{2,1}}
\end{array}\right\} \Rightarrow\left(\frac{L_{1}}{L_{2}}\right)^{2}
\end{aligned}
$$

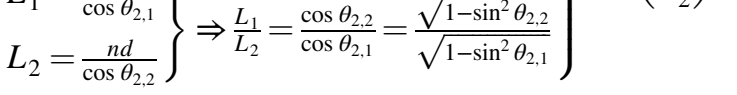

$$
\begin{aligned}
& =\frac{n^{2}-\sin ^{2} \theta_{2,1}}{n^{2}-\sin ^{2} \theta_{1,1}} \Rightarrow L_{1}^{2}\left(n^{2}-\sin ^{2} \theta_{1,1}\right)=L_{2}^{2}\left(n^{2}-\sin ^{2} \theta_{2,1}\right) \Rightarrow \\
& n=\sqrt{\frac{L_{1}^{2} \sin \left(\theta_{1,1}\right)-L_{2}^{2} \sin \left(\theta_{1,2}\right)}{L_{1}^{2}-L_{2}^{2}}}
\end{aligned}
$$

The path lengths $\left(L_{1}\right.$ and $\left.L_{2}\right)$ can be measured directly from the images, and the incidence angles $\left(\theta_{1,1}, \theta_{1,2}\right)$ can also be measured directly from the angle of the top surface of the sample. This approach was verified using samples placed over a reflector so that the path-length change could be easily measured and, at the same time, use the same reflector as a reference for estimating $n$ using the technique described by Tearney et al. ${ }^{9}$

However, distinct, highly reflective, structures are rarely present in tissue. To apply this technique in vivo, a different approach is required to estimate the path-length change. This can be achieved by first registering and aligning the two images, with a rigid affine transformation, and subsequently using the cross-correlation of corresponding A-scans from each image to estimate the path-length change for each A-scan. Affine registration is an intensity-based linear mapping technique that secures points, straight lines, and planes. It consists of scaling, rotation, and translation, and all pixels go through the same transformation. It is, usually, used to improve geometric distortions such as differences in image's size. The affine transformation can be described as

$$
\begin{aligned}
& x^{\prime}=t_{x}+a_{1} x+a_{2} y \\
& y^{\prime}=t_{y}+b_{1} x+b_{2} y
\end{aligned}
$$

where $t_{x}$ and $t_{y}$ refer to the translation vectors; $x^{\prime}$ and $y^{\prime}$ are the original points; and $a_{1}, a_{2}, b_{1}$, and $b_{2}$ are the affine transformation parameters. ${ }^{17}$

After affine registration, the images are matched in rotation and translation. However, the second image is distorted, in depth, relative to the first because of the elongation of the path lengths due to the larger angle of incidence [Fig. 3(b)]. The cross-correlation is, therefore, expected to have maxima, first, at the 0 lag and then, again, at the lag, which depends on the magnitude of this distortion. The path-length change, $\Delta L$, is estimated from the lag of this second maximum of the cross-correlation [Fig. 3(c)]. Since the path length of the second image,

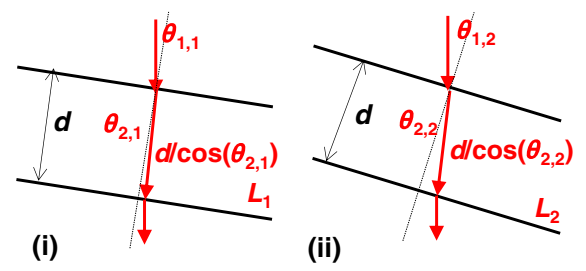

(b) (ii)

(a)

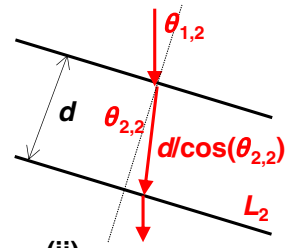

(ii)

Fig. 2 Incidence angles and path lengths of beams perpendicular and at an angle to the samples. (a) Perpendicular (i) and at an angle (ii) incidence on the sample. (b) Two samples with different incidence angles $\theta_{1,1}$ (i) and $\theta_{1,2}$ (ii). 


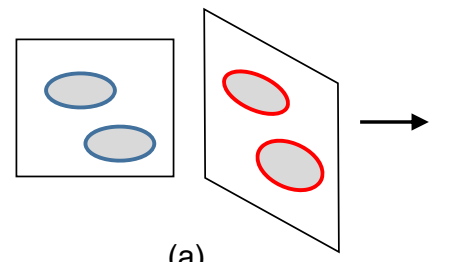

(a)

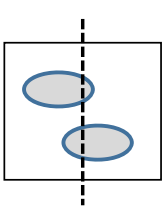

(b)

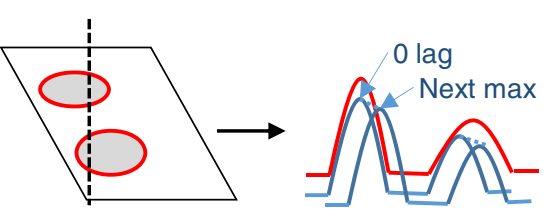

(c)

Fig. 3 Path-length change estimate from two OCT images taken at different incidence angles. The images are first (a) registered and (b) aligned. (c) The cross-correlation of corresponding A-scans from the aligned images exhibit distinct maxima.

$L_{2}$, can now be calculated by the path-length change, i.e., $L_{2}=L_{1}+\Delta L$, the index is, then, calculated by Eq. (2):

$$
n=\sqrt{\frac{L_{1}^{2} \sin \left(\theta_{1,1}\right)-\left(L_{1}+\Delta L\right)^{2} \sin \left(\theta_{1,2}\right)}{L_{1}^{2}-\left(L_{1}+\Delta L\right)^{2}}} .
$$

\subsection{Experimental Methods}

A swept source OCT system, with $1.3-\mu \mathrm{m}$ center wavelength and $12-\mu$ m resolution in air (Santec IVS 300), was used to image samples of glass, cucumber, and rabbit trachea. The system operated at an A-scan rate of $20 \mathrm{kHz}$ and collected raw interferometric data corresponding to a physical size of $5 \times 5 \mathrm{~mm}$ $(512 \times 2018$ pixels $)$. Each sample was placed on a vertically mounted rotation stage (0.01-deg accuracy), which was rotated manually using a micrometer. For each sample, $n$ and the sample thickness $(L)$ were measured using the Tearney et al. ${ }^{9}$ method, which served as the reference value. Subsequently, $n$ was estimated from the path-length difference of the dual-angle images.
To verify the technique, the mirror below the sample was initially used to measure the path-length changes. To prove the applicability of the technique to in vivo tissue imaging, the index of refraction was also calculated with the cross-correlation lag method. In this case, no mirror reflection was used. All methods were applied to the same sample images to ensure that the results were comparable. The applicability of the proposed method was evaluated, first, on uniform, nonscattering, glass and, subsequently, higher scattering samples (cucumber) with more complicated structures and varying thicknesses and angles of incidence. The accuracy of the results was evaluated relative to the reference values. Images of glass, cucumber slices, and freshly excised rabbit trachea were acquired at different incidence angles, ranging from $5 \mathrm{deg}$ to $20 \mathrm{deg}$, with eight repetitions for each combination.

\section{Results}

Initially, the proposed methodology was tested on glass. Images of glass were acquired at different incidence angles, ranging

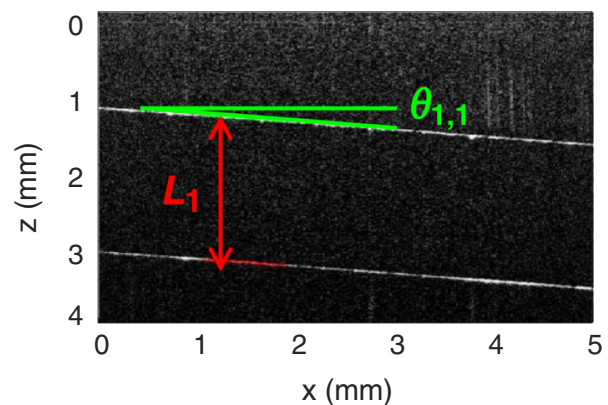

(a)

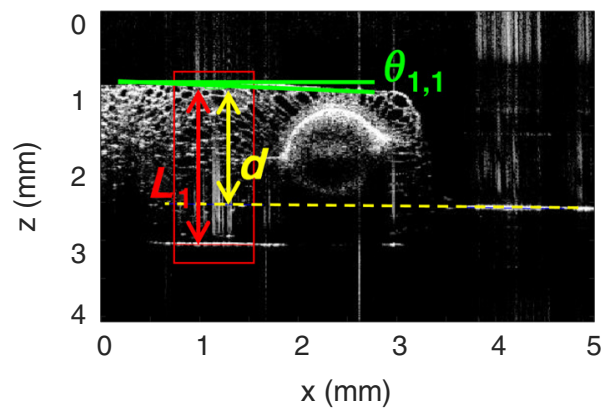

(c)

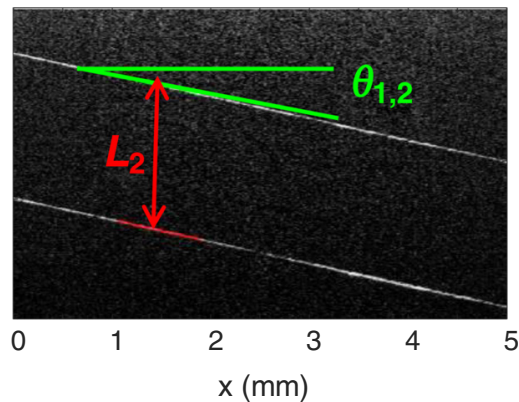

(b)

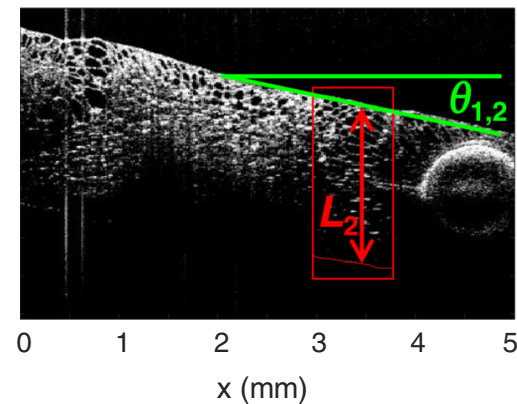

(d)

Fig. 4. (a), (b) Glass. (c), (d) Cucumber. The angles $\theta_{1,1}$ and $\theta_{1,2}$ for the glass were 4.5 deg and $11.8 \mathrm{deg}$, respectively, whereas for the cucumber, those angles were 0.9 deg and 12.2 deg, respectively. 
Table 1 Index of refraction estimates.

\begin{tabular}{|c|c|c|c|c|c|c|c|c|c|c|}
\hline & \multicolumn{2}{|c|}{ Reference } & \multicolumn{4}{|c|}{$\begin{array}{c}\text { Path-length changes measured } \\
\text { using a reflector }\end{array}$} & \multicolumn{4}{|c|}{$\begin{array}{c}\text { Path-length changes measured } \\
\text { using Xcorr }\end{array}$} \\
\hline & Mean & Std & Mean & Std & Error & $\%$ Error & Mean & Std & Error & \% Error \\
\hline Glass & $1.509^{a}$ & $\mathrm{~N} / \mathrm{A}$ & 1.530 & 0.091 & 0.021 & 1.36 & - & - & - & - \\
\hline Cucumber & $1.369^{b}$ & 0.015 & 1.379 & 0.025 & 0.009 & 0.688 & 1.354 & 0.024 & 0.015 & 1.097 \\
\hline Rabbit trachea & $1.391^{b}$ & 0.002 & 1.390 & 0.001 & 0.001 & 0.001 & 1.392 & 0.002 & 0.001 & 0.001 \\
\hline
\end{tabular}

${ }^{\text {aP }}$ rovided by the manufacturer.

${ }^{\mathrm{b}}$ Measured using the method of Tearney et al. ${ }^{9}$

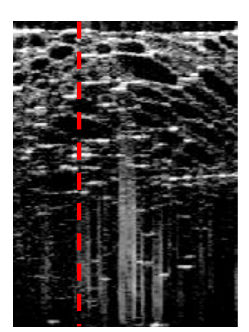

(a)

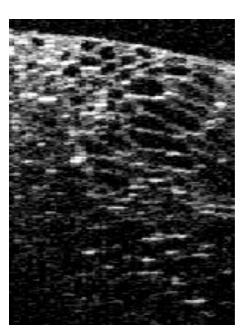

(b)

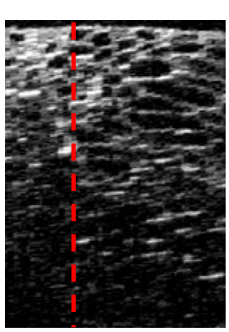

(c)

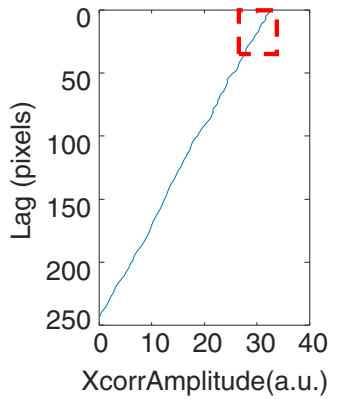

(d)

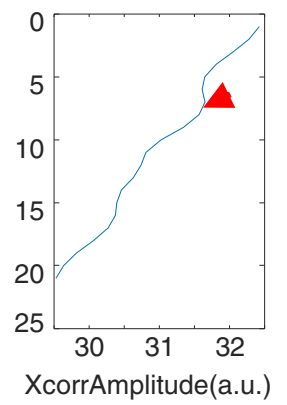

(e)

Fig. 5 (a), (b) Portions of the original OCT images of cucumber (Fig. 4), at different incidence angles. (c) The registration of (b) on (a). (d) The cross-correlation of the A-scans indicated by the dashed lines. (e) A zoomed region (indicated by the dashed box) of (d). The arrow points to the first maximum after the zero lag.

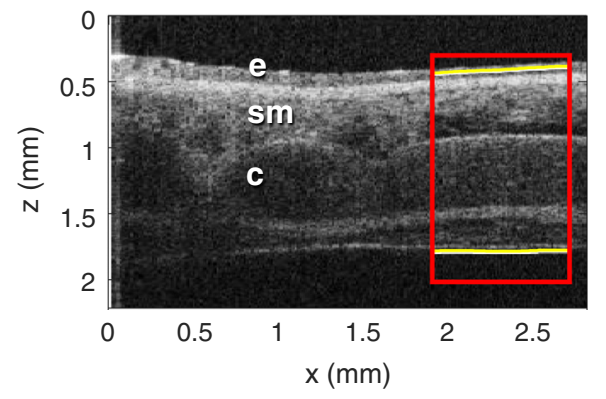

(a)

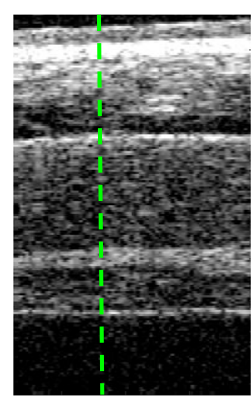

(c)

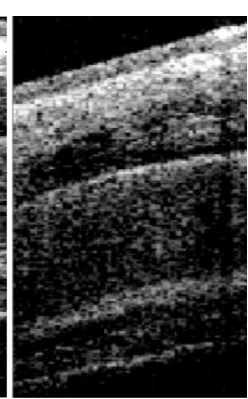

(d)

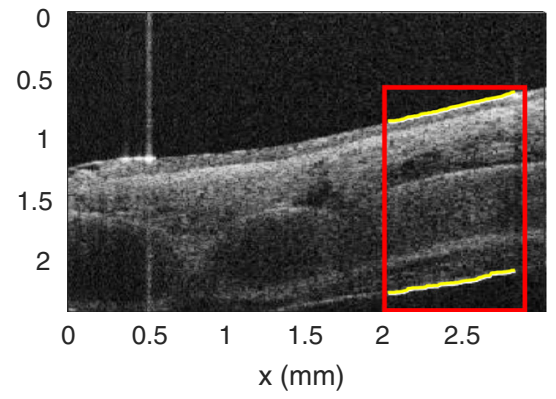

(b)

Fig. 6 (a), (b) The original OCT images of freshly excised trachea, at different incidence angles. The labels refer to the epithelium (e), the submucosa (sm), and cartilage (c). (c) The portion of (a). (d) Portion of (b). (e) The registered version of (d). (f) The cross-correlation of the A-scans indicated by the dashed lines in (c) and (e). The arrow points to the first maximum after the zero lag (not shown here for better visualization of the peaks). 
from 5 deg to $20 \mathrm{deg}$, with eight repetitions for each combination, for a total of 48 images [Figs. 4(a) and 4(b)]. The average results are shown in Table 1 . When compared to the reference value of the index of the particular glass, $n=1.509$, the error was $1.36 \%$. The accuracy and precision of the path-length measurements were also evaluated from the glass measurements.

Imaging of thin cucumber slices followed, in an effort to examine the validity of the method in higher scattering samples [Figs. 4(c) and 4(d)]. The average results, when using the reflector below the sample to measure the path lengths, ${ }^{9}$ are also shown in Table 1 . The estimated $n$, compared to the reference value, exhibited a mean error of $\sim 0.7 \%$ (varying from $0.5 \%$ to $2 \%$ ). The index of refraction was also determined using the cross-correlation method (Fig. 5). The results are also shown in Table 1 . When compared to the reference value, the error was $\sim 1.1 \%$ (varying from $0.1 \%$ to $3 \%$ ). The precision of the measurements of angle and distance were also evaluated from the OCT images of glass. When measuring the angle, from various locations within the same image, the values did not vary by more than a standard deviation of $0.7 \mathrm{deg}$. In addition, the measured path length, within the same image, varied by $<8 \mu \mathrm{m}$ standard deviation. The accuracy of the measurements was evaluated by comparing to the expected path length, i.e., $L=n d / \cos \left\{a \sin \left[\frac{\sin \left(\theta_{1}\right)}{n}\right]\right\}$, and was found not to vary by more than $9 \mu \mathrm{m}$.

Finally, to validate the applicability of the proposed technique to tissue samples, freshly excised rabbit trachea was imaged. The results are shown in Fig. 6 and Table 1. The proposed methods (measuring the path-length change with or without reflector). ${ }^{9}$

The variation in the error, reaching in some cases $3 \%$, is explained by but also exemplifies the most serious limitation of the proposed technique, i.e., image misalignment. Misalignment by as little as $\pm 10 \mu \mathrm{m}$ between the two images could lead to up to $5 \%$ error, as measured experimentally. This limitation should be taken into account and alignment should be carefully monitored especially when measuring the index of nonuniform samples. Fortunately, angle-resolved OCT systems, which are specifically designed to image the same sample area under different incident angles, can provide angular and spatial precisions limited only by the precision of the galvanometric scanners. The performance of such systems has been validated for speckle reduction and results indicate that there is no resolution degradation implying negligible spatial shifts. ${ }^{18}$

\section{Conclusions}

In this paper, we propose a measurement technique that could be deployed for in vivo estimation of $n$. This technique uses two OCT images obtained at different incidence angles and estimates $n$ by measuring the path-length changes and the incidence angles. In tissues with no distinct reflectors, the path-length change can be estimated by first registering and aligning the two images, with a rigid affine transformation, and subsequently using the cross-correlation of corresponding A-scans from each image. Experimental verification produced values of $n$ with a mean error of $\sim 1 \%$ compared to the expected values. The main limitation of this technique is the effect of misalignment of the images on the results, which can result in up to $5 \%$ error for $10 \mu \mathrm{m}$ misalignment. However, the use of an angular-resolved system should provide the precision required for the application of the proposed technique. These initial results are promising and provide evidence that this method should be further investigated and validated on human tissues so that, in the future, it could be developed into a clinically useful diagnostic tool.

\section{Disclosures}

The authors have no relevant financial interests in this article and no potential conflicts of interest to disclose.

\section{Acknowledgments}

This study was funded by the European Union's Horizon 2020 research and innovation program under Grant Agreement No. 739551 (KIOS CoE) and from the Republic of Cyprus through the Directorate General for European Programmes, Coordination and Development.

\section{References}

1. J. Izatt et al., "Optical coherence microscopy in scattering media," Opt. Lett. 19(8), 590-592 (1994).

2. M. Choma, A. K. Ellerbee, and C. Yang, "Spectral-domain phase microscopy," Opt. Lett. 30(10), 1162-1164 (2005).

3. Y. Cotte et al., "Marker-free phase nanoscopy," Nat. Photonics 7(2), 113-117 (2013).

4. T. Kim et al., "White-light diffraction tomography of unlabelled live cells," Nat. Photonics 8(3), 256-263 (2014).

5. D. J. Weatherall, "Systems biology and red cells," N. Engl. J. Med. 364(4), 376-377 (2011).

6. H. Onodera, I. Awal, and J. Ikenoue, "Refractive-index measurement of bulk materials: prism coupling method," Appl. Opt. 22(8), 1194-1197 (1983).

7. A. J. Werner, "Methods in high precision refractometry of optical glasses," Appl. Opt. 7(5), 837-843 (1967).

8. H. J. Choi et al., "Measurement of refractive index and thickness of transparent plate by dual-wavelength interference," Opt. Express 18(9), 9429-9434 (2010).

9. G. J. Tearney et al., "Determination of the refractive index of highly scattering human tissue by optical coherence tomography," Opt. Lett. 20, 2258-2260 (1995).

10. X. Wang et al., "Simultaneous refractive index and thickness measurements of bio-tissue by optical coherence tomography," J. Biomed. Opt. 7(4), 628-632 (2002).

11. Y. Verma et al., "Measurement of gradient refractive index profile of crystalline lens of fisheye in vivo using OCT," Appl. Phys. B 87(4), 607-610 (2007).

12. S. R. Uhlhorn et al., "Refractive index measurement of the isolated crystalline lens using optical coherence tomography," Vision Res. 48(27), 2732-2738 (2008).

13. R. Chakraborty et al., "Refractive index measurement of the mouse crystalline lens using optical coherence tomography," Exp. Eye Res. 125, 62-70 (2014).

14. P. H. Tomlins et al., "Optical coherence refractometry," Opt. Lett. 33, 2272-2274 (2008).

15. P. Rajai et al., "Simultaneous measurement of refractive index and thickness of multilayer systems using Fourier domain optical coherence tomography, part 1: theory," J. Biomed. Opt. 22(1), 015002 (2017).

16. Z. Turani et al., "Refractive index correction in optical coherence tomography images of multilayer tissues," J. Biomed. Opt. 23(7), 070501 (2018).

17. Y. Diez et al., "Revisiting intensity-based image registration applied to mammography," IEEE Trans. Inf. Technol. Biomed. 15(5), 716-725 (2011).

18. A. E. Desjardins et al., "Angle-resolved optical coherence tomography with sequential angular selectivity for speckle reduction," Opt. Express. 15(10), 6200-6209 (2007).

Christos Photiou received his BSc degree in physics from the University of Patras and his MSc degree in environmental health from the Cyprus University of Technology (in association with the Harvard School of Public Health). He is currently a PhD student in the Department of Electrical and Computer Engineering at the University of Cyprus and a researcher at the KIOS Research and 
Photiou and Pitris: Dual-angle optical coherence tomography for index of refraction estimation using rigid registration and cross-correlation

Innovation Center of Excellence. His research interests include biomedical image processing, machine learning, and optical diagnostics.

Costas Pitris is an associate professor in the Department of Electrical and Computer Engineering and the KIOS Research and
Innovation Center of Excellence at the University of Cyprus. He holds BS and MS degrees from the University of Texas at Austin, a PhD from the Massachusetts Institute of Technology, and an MD from the Harvard Medical School. His main research interests are in the area of biomedical optics applied to medical diagnosis and monitoring. 